\title{
Entrapment Neuropathies of the Lower Extremity
}

\section{Anita Craig, DO}

Abstract: Neuropathies that affect the lower limbs are often encountered after trauma or iatrogenic injury or by entrapment at areas of anatomic restriction. Symptoms may initially be masked by concomitant trauma or recovery from surgical procedures. The nerves that serve the lower extremities arise from the lumbosacral plexus, formed by the L2-S2 nerve roots. The major nerves that supply the lower extremities are the femoral, obturator, lateral femoral cutaneous, and the peroneal (fibular) and tibial, which arise from the sciatic nerve, and the superior and inferior gluteal nerves. An understanding of the motor and sensory functions of these nerves is critical in recognizing and localizing nerve injury. Electrodiagnostic studies are an important diagnostic tool. A well-designed electromyography study can help confirm and localize a nerve lesion, assess severity, and evaluate for other peripheral nerve lesions, such as plexopathy or radiculopathy.

PM R 2013;5:S31-S40

\section{INTRODUCTION}

Neuropathies of the lower extremities frequently accompany trauma or surgical interventions to the abdomen, pelvis, and lower limbs. Initial presentation of these neuropathies may be masked by acute injuries and may be appreciated only when functional limitation persists beyond the expected time course of recovery from the initial injury. Lower limb neuropathies may profoundly affect mobility. The initial approach to suspected neurologic injury involves a careful physical examination to characterize the distribution of motor and sensory loss. The role of electromyography is to confirm and localize the neurologic injury, assess severity of the injury to allow prognostication, aid in forming a management plan, and exclude confounding or coexisting neurologic injury.

\section{FEMORAL NEUROPATHY}

The femoral nerve arises from the second through fourth lumbar spinal nerve roots and travels between the psoas and iliacus muscles. After innervating these muscles within the pelvis, it passes under the inguinal ligament lateral to the femoral artery and vein. The nerve then divides into its terminal branches, with muscular branches supplying the 4 heads of the quadriceps muscle, sartorius, and pectineus, with medial and intermediate sensory branches to the anterior thigh, and the saphenous nerve supplying cutaneous innervation to the medial leg (Figure 1).

The femoral nerve is most commonly injured within the retroperitoneal space or under the inguinal ligament. The most frequent cause of femoral neuropathy is iatrogenic injury during intra-abdominal and pelvic surgical procedures as well as gynecologic and urologic operations. Hip procedures have also been implicated, especially with anterior or anterolateral approaches, potentially from several mechanisms. Direct compression of the nerve may occur from the use of self-retaining retractors [1]. Positioning in the lithotomy position can compromise the nerve at the inguinal ligament due to sharp angulation of the nerve in the flexed, abducted, and externally rotated position [2]. The nerve may also be injured due to ischemia from iliac vessel compression or laceration from trocar insertion during laparoscopic surgery. Hematomas in the setting of anticoagulation and blood dyscrasias, particularly in the setting of femoral catheterization procedures, are another common cause of femoral neuropathy [3].

A.C. University of Michigan, 325 E. Eisenhower, Ann Arbor, Ml 48108. Address correspondence to: A.C.; e-mail: aswcraig@med. umich.edu

Disclosure: nothing to disclose 


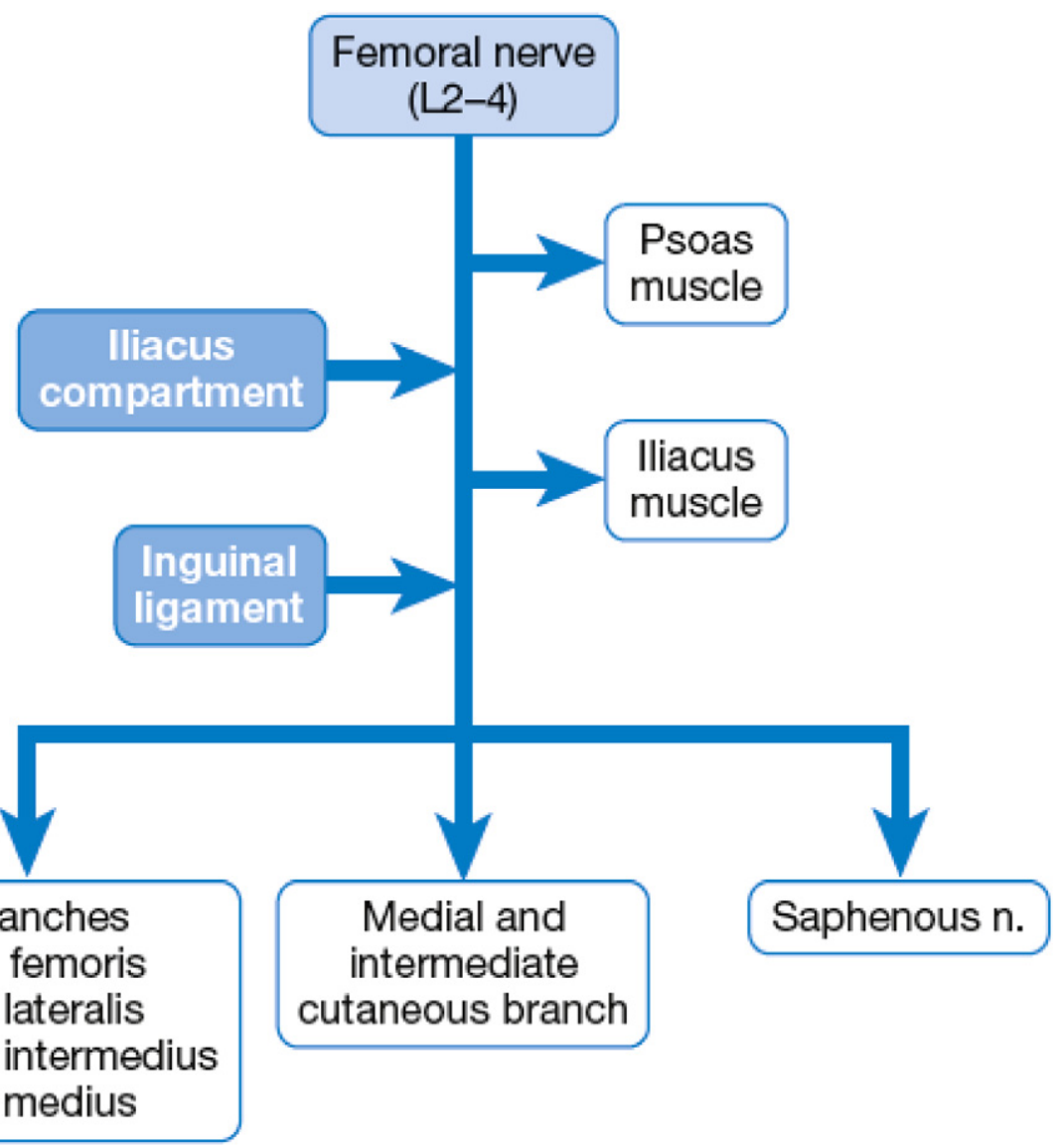

Figure 1. Femoral nerve. (Reprinted with permission from Craig A, Richardson J. Rehabilitation of patients with neuropathies. In: Braddom RL, ed. Physical Medicine and Rehabilitation. Philadelphia, PA: Elsevier; 2011, 1065-1095) (25).

Individuals with femoral neuropathy will present with unilateral weakness of knee extension and a feeling of instability around the knee. Occasionally, bilateral lesions can occur after lithotomy positioning. Hip flexion will be weak in lesions that occur proximal to the inguinal ligament due to involvement of the iliopsoas, or atrophy of the quadriceps may be appreciated. From a functional standpoint, stairs and inclines may be particularly problematic. The patellar reflex is usually depressed or absent, and sensory loss can be seen in the anterior thigh and lower leg in the saphenous nerve distribution. Hip adduction and abduction, knee flexion, and distal strength are preserved. Pain may be elicited by extension of the hip.

Electrodiagnostic testing can be helpful in localizing the lesion to proximal or distal to the inguinal ligament and to rule out other processes that can present with proximal thigh weakness, specifically high lumbar plexopathy or radiculopathy. Nerve conduction studies (NCS) of the femoral nerve can be obtained by stimulating the femoral nerve at the inguinal ligament and recording over the quadriceps. This study can be particularly uncomfortable because of the longer-duration stimulus that may be required, due to the depth of the nerve. Comparison should be made with the clinically normal opposite side. Obtaining a compound action potential of at least $50 \%$ of the other side suggests good prognosis for recovery within 1 year [4]. The needle examination should include both the quadriceps and iliopsoas. Abnormalities in the iliopsoas localize the nerve lesion to within the pelvis. Sampling of the adductor and paraspinal muscles is useful for excluding a high lumbar radiculopathy or plexopathy.

\section{Saphenous Neuropathy}

The saphenous nerve is the purely sensory terminal branch of the femoral nerve. It traverses the quadriceps muscle within the subsartorial (Hunter) canal that accompanies the femoral artery. It exits the canal $10 \mathrm{~cm}$ above the knee, piercing a fascial layer where it gives off an infrapatellar branch that supplies the knee. The nerve then descends along the medial surface of the tibia and medial malleolus, which supplies sensation to the medial aspect of the lower leg and medial foot as far as the first metatarsal phalangeal joint.

The saphenous nerve can be injured at various points in its course. In the thigh, it travels with the femoral artery and 
therefore can be vulnerable to procedures that involve the artery, such as catheterization and thrombectomy. The nerve can also be injured as it exits the subsartorial canal, particularly in patients with genu valgus and internal tibial torsion, because the nerve can be sharply angulated as it passes through the fascial layer. Nerve lesions at the level of the knee have been reported with knee arthroscopy and meniscectomy [5]. Knee orthoses can cause external compression at the knee. Isolated lesions of the infrapatellar branch can also be seen. In the lower leg, the nerve travels adjacent to the saphenous vein and can be injured in varicose vein stripping as well as during harvesting the saphenous vein for grafting [6].

Clinical presentation of saphenous neuropathy may range from minimal sensory loss in the medial lower leg to severe neuropathic pain. Isolated injury to the infrapatellar branch may present with medial knee pain that may be mistaken for medial knee compartment pathology. Occasionally, a neuroma may be palpated or the Tinel sign is elicited along the course of the nerve. A pure saphenous nerve injury should not produce any weakness.

In cases of suspected saphenous nerve injury, corresponding NCS can be performed, although these are technically difficult and responses can be very small; values should be compared with the unaffected side. Results of a needle examination should be normal and are useful to rule out femoral neuropathy, L4 radiculopathy, or a lumbar plexopathy.

\section{Obturator Neuropathy}

The obturator nerve is formed from the L2-4 nerve roots. It forms within the psoas and runs anterior to the sacroiliac joint and innervates the adductor muscles of the thigh. The anterior branch supplies the adductor brevis and longus and gracilis muscles and sensory innervation to the medial thigh. The posterior branch innervates the obturator externus and a portion of the adductor magnus, which is also supplied by the sciatic nerve. Sensory branches also contribute to sensation to the knee joint.

Isolated damage to the obturator nerve is infrequent but may arise from direct trauma due to pelvic fractures, particularly those that affect the sacroiliac joint, although the extent of these injuries will usually involve other nerves, the plexus, or spinal roots. Total hip arthroplasty, pelvic operations, and femoral artery procedures, as well as compression from tumor or metastatic disease, have also been implicated [7]. Obturator injury has been associated with lithotomy positioning [8]. Prolonged labor may injure the nerve due to compression of the fetal head against the lateral wall of the pelvis [9]. The nerve may also be entrapped as it enters the thigh in the adductor canal. This often will present as pain induced by exercise [10].

Patients with obturator neuropathy will most often present with pain and sensory loss in the groin or medial thigh.
When following surgery or pelvic trauma, symptoms of pain from obturator injury may be masked or difficult to differentiate from somatic pain from the pelvic injuries. Weakness of adduction and internal rotation of the leg may be seen and functionally may present as a circumducted gait.

There is no NCS available to assess the obturator nerve. Needle electromyography is helpful in confirming suspected obturator neuropathy with abnormalities in the adductor muscles. If evaluating the adductor magnus, it is important to recognize that the sciatic nerve also contributes to its motor innervation. Assessment of femoral-innervated muscles and paraspinal muscles should be performed to rule out plexus injury or radiculopathy.

\section{LATERAL FEMORAL CUTANEOUS NEUROPATHY}

The lateral femoral cutaneous is a purely sensory nerve formed from the second and third lumbar nerves. It emerges from the lateral border of the psoas and crosses the iliacus muscle before emerging from the pelvis medial to the anterior superior iliac spine (ASIS) under the inguinal ligament. The nerve is especially vulnerable to injury in this area. It then divides into anterior and posterior branches and supplies the sensory territory of the anterolateral thigh.

Injury of the nerve, which leads to the clinical picture of meralgia parasthetica, most frequently arises as the nerve passes under the inguinal ligament near the ASIS. At this location, it is vulnerable to compression from external sources, such as tool belts, seat belts, or tight waist bands. Obesity and pregnancy as well as rapid weight loss have all been implicated in meralgia parasthetica [11]. The nerve can also be injured during surgical procedures, such as inguinal hernia repair, iliac bone graft harvesting, renal transplantation, and hip surgery [12]. Tumor can compress the nerve at the iliac crest. Within the pelvis, the nerve can be affected by pelvic masses such as abdominal aneurysm or neoplasm. Direct blows to the iliac crest can also cause injury to this nerve.

Meralgia parasthetica is characterized by sensory loss and paresthesias in a discrete distribution in the anterolateral thigh. Pain may be exacerbated by extension of the leg, usually worse with standing and walking, and better with sitting or flexing the hip. Compression over the ASIS may also worsen symptoms, with waistbands or even undergarments aggravating the pain. A Tinel sign may be present with percussion over the ASIS. There should be no loss of motor strength in this purely sensory neuropathy, and the patellar reflexes are preserved.

Lateral femoral cutaneous neuropathy can be confirmed electrodiagnostically by performing NCS and comparing with the unaffected side. This, however, is a technically difficult response to obtain even in normal people with average body habitus [13]. Ultrasound can be used to identify 
nerve location and improves the ability to record sensory nerve action potentials [14]. Electromyography is most useful to exclude other conditions, such as L2 radiculopathy or high lumbar plexopathy or femoral neuropathy.

\section{Peroneal (Fibular) Neuropathy}

Fibers from the L4-S1 nerve roots form the common peroneal nerve, which runs with the tibial nerve as part of the sciatic in the posterior thigh. The peroneal nerve diverges at the level of the popliteal fossa after giving off a muscular branch to the short head of the bicep femoris, which is the only muscle that it supplies in the thigh. Within the popliteal fossa, it gives off a sensory contribution that joins with a branch from the tibial to form the sural nerve. The common peroneal nerve wraps around the fibular head and neck, where it closely adheres to the periosteum and is very vulnerable to both compression and trauma. It then divides into its terminal branches, the superficial and deep peroneal nerves. The superficial peroneal portion supplies muscular branches to the peroneus longus and brevis and sensory innervation to the lower two-thirds of the lateral leg and the dorsum of the foot, with the exception of the first web space. The deep peroneal branch enters the anterior compartment of the leg to supply the anterior tibialis, extensor hallucis longus, peroneus tertius, and extensor digitorum brevis (EDB) muscles, and sensory innervation to the first web space. An important anatomic variant to recognize is the accessory peroneal nerve from the superficial peroneal nerve, which contributes innervation to the EDB muscle in approximately one-third of the population (Figure 2) [15].

Peroneal mononeuropathies are the most common mononeuropathy in the lower extremity. The common peroneal nerve can be injured due to acute trauma, commonly as it passes around the fibular head. Due to its superficial nature, it can be easily injured by direct blows or lacerations in this area. It is vulnerable to fracture and dislocation of the fibular head and neck due to their anatomically intimate relationship. Severe flexion and inversion sprains at the ankle can cause traction injuries due to tearing of the vasa nervorum [16]. Probably the most common cause of peroneal neuropathy is external compression of the nerve at the fibular head, which can occur with habitual knee crossers or in people who squat or kneel for extended periods of time, such as carpet layers or farm workers ("strawberry pickers' palsy"). It is also seen in bedridden patients due to either positioning or weight loss. Casts and braces can also cause direct compression. This neuropathy may also arise from lithotomy positioning but not as commonly as other neuropathies discussed earlier. Baker cysts, neuromas, callus formation from fibular fractures, and tumors can cause internal compression injuries. Isolated injury to the deep branch can occur from the pressure effects of anterior compartment syndrome.

Patients with lesions to the peroneal nerve will present with partial or complete foot drop, with weakness of the ankle dorsiflexors and evertors, and with preservation of plantarflexion and inversion. Gait may be characterized by foot slap or a steppage gait as the patient attempts to clear the foot. More subtle injuries may be noticed as a propensity to "stub the toe." Sensory loss may be seen in the anterolateral surface of the lower leg or preferentially on the dorsum of the foot in incomplete lesions. Tenderness and/or a Tinel sign may be provoked with percussion around the fibular head; reflexes should be spared.

NCS can be helpful in localizing a peroneal lesion. Peroneal motor conduction to the EDB should be performed, stimulating both below and above the fibular head. There are, however, important limitations to recognize. First, this muscle can be denervated due to local trauma or in the setting of distal neuropathy. In addition, in one-third of the population, an accessory peroneal nerve that arises from the superficial peroneal nerve can contribute to the EDB. This variant can be recognized when the amplitude obtained when stimulating above the fibular head is greater than when stimulating below this area and is verified by obtaining a response stimulating behind the lateral malleolus (Figures 3 and 4). Peroneal motor conduction can also be obtained from the anterior tibialis. Comparison with the opposite side is helpful in quantifying the degree of axon loss. Reduction of the superficial peroneal sensory response indicates a lesion distal to the dorsal root ganglia. Tibial motor, F-response, and sural nerve studies are useful in ruling out a peripheral neuropathy or a more widespread lesion, such as a plexopathy or sciatic injury (Table 1). Needle electromyography helps to further localize the lesion, rule out other causes of foot drop, and assess the severity of the axon loss. Muscles from both the superficial and deep branch of the peroneal nerve should be sampled. If these are abnormal, then sampling of the short head of the biceps femoris will help in localizing the lesion to above or below the knee, because this muscle is the only peroneal-innervated muscle above the knee. Studying tibialinnervated muscles can assess for the presence of a sciatic lesion, and an L5-supplied muscle not from the peroneal nerve (tibialis posterior or gluteus medius) will aid in excluding an L5 radiculopathy. If more widespread abnormalities are found, then sampling of the lumbar paraspinal muscles will help differentiate plexus versus root level lesions (Table 2).

\section{Tibial Neuropathies}

The tibial division of the sciatic nerve is formed by L5-S2 nerve roots and, as part of the sciatic nerve, supplies all of the hamstring muscles with the exception of the short head of the biceps femoris. It also provides partial innervation to the adductor magnus. Within the popliteal fossa, it contributes to the sural nerve and supplies the muscles of the posterior compartment of the lower leg. It then passes posterior to the medial malleolus through the tarsal tunnel and then divides into its terminal branches, the calcaneal, medial plantar, and lateral plantar (Figure 5). 


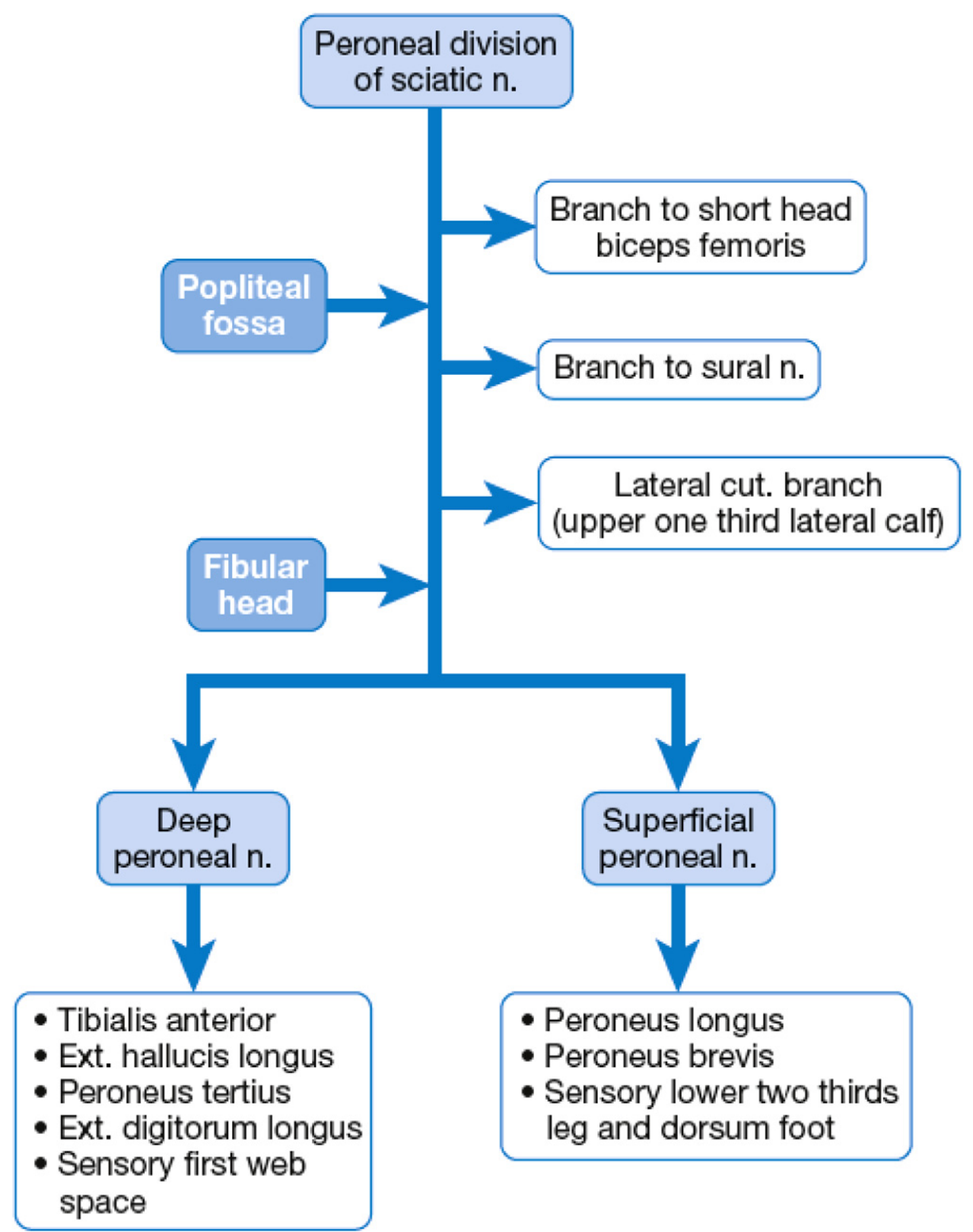

Figure 2. Peroneal nerve (Reprinted with permission from Craig A, Richardson J. Rehabilitation of patients with neuropathies. In: Braddom RL, ed. Physical Medicine and Rehabilitation. Philadelphia, PA: Elsevier; 201 1, 1065-1095) (25).

Because the tibial nerve runs deep within the popliteal space and the posterior leg, it is relatively well protected and infrequently injured compared with the more superficially located peroneal nerve. Proximal injury at the level of the knee may occur due to space-occupying lesions in the popliteal fossa, such as Baker cysts or hemorrhage. Distal injury at the level of the ankle occurs under the flexor retinaculum and is commonly referred to as tarsal tunnel syndrome. The majority of lesions at this level are due to idiopathic compression but may also arise from ankle trauma; masses from lipomas or ganglion cysts; or heel or foot deformity, for example, talocalcaneal coalition [17].

Proximal tibial lesions cause weakness of plantarflexion and inversion of the ankle and loss of toe flexion strength with accompanying atrophy of the calf. Ankle dorsiflexion and knee flexion should be preserved. With incomplete lesions, weakness may be subtle and easily missed, particularly in the medial gastrocnemius. To detect subtle weakness, this muscle should be tested by having the patient perform repeated unipedal toe raises. Sensory loss can be seen in the 


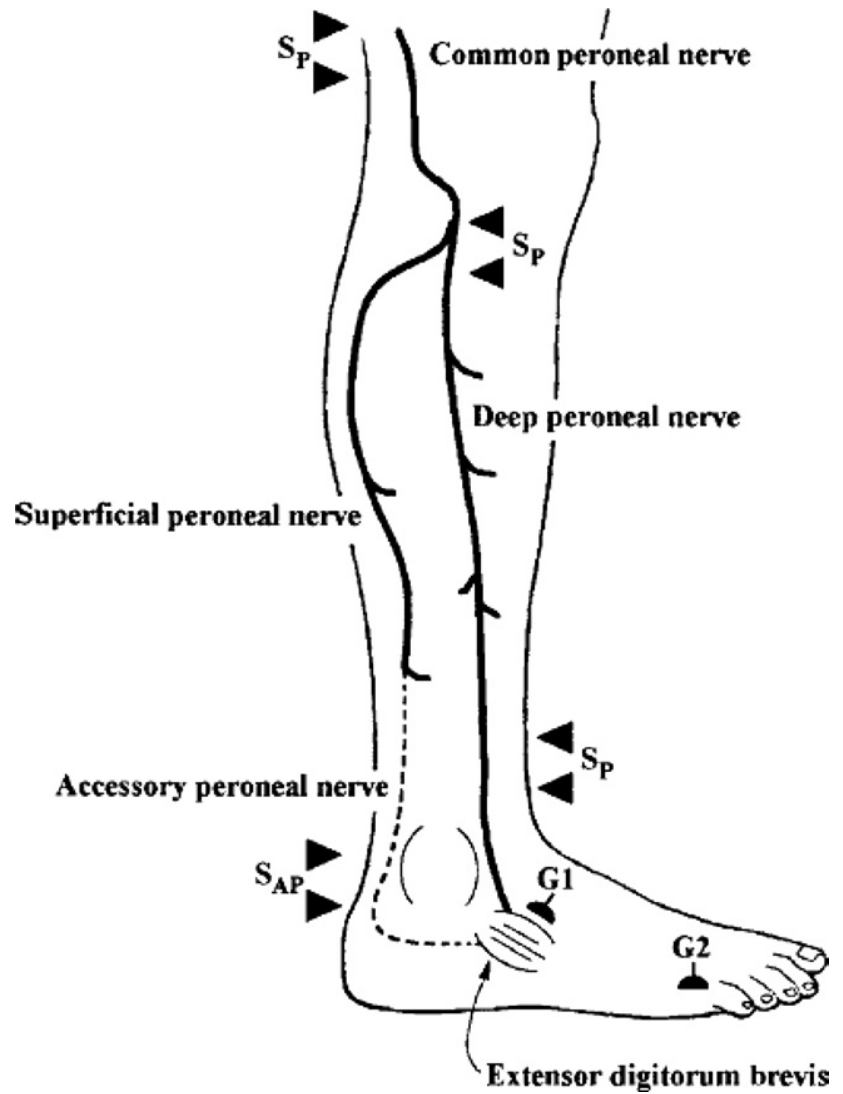

Figure 3. Standard peroneal motor nerve conduction studies are performed by stimulating at the anterior ankle $\left(S_{p}\right)$ while recording over the extensor digitorum brevis (EDB). To assess for an accessory peroneal nerve (APN), the stimulator is placed posterior to the lateral malleolus $\left(\mathrm{S}_{\mathrm{AP}}\right)$ while recording over the EDB. (Reprinted with permission from Preston DC, Shapiro BE. Anomalous innervations. In: Preston DC, Shapiro BE. Electromyography and Neuromuscular Diseases. 2nd ed. Philadelphia, PA: Elsevier Butterworth Heinemann; 2005, 79-86) (26).

sole of the foot and the posterolateral lower leg and foot in the sural distribution. Loss of sural sensation may be variable because this nerve also receives peroneal contribution. The Achilles reflex is also depressed or absent. In lesions at the mid lower leg, loss of toe flexor strength may be noted with preservation of ankle strength. Tarsal tunnel syndrome presents with numbness and pain in the sole of the foot, often sparing the calcaneal branch to the heel. A Tinel sign can sometimes be elicited by tapping over the flexor retinaculum at the medial malleolus.

Electrodiagnostic evaluation of suspected tibial lesions should include sural sensory responses, which will be variably affected in proximal tibial lesions. Comparison with the unaffected side may demonstrate asymmetry, which can be helpful with borderline normal responses. Reduction in tibial motor responses to the abductor hallucis will be seen. In cases of suspected tarsal tunnel syndrome, medial and lateral plantar responses should be performed. These can be difficult to obtain, particularly in the setting of thick calluses or preexisting peripheral neuropathy. Comparison with the unaffected side should be performed before drawing any conclusions based on low or absent responses. Needle electromyography will demonstrate denervation in the medial gastrocnemius or posterior tibialis in proximal tibial lesions. Mid leg lesions may spare these muscles, which affect the long toe flexors and foot intrinsics only. In suspected tarsal tunnel syndrome, examining the first dorsal interosseous muscle reflects injury of the lateral plantar branch and the adductor hallucis, the medial plantar branch. Abnormalities of these muscles are also seen in early peripheral neuropathies; therefore, comparison with the contralateral side is recommended. Differential diagnosis of tibial neuropathy includes S1 radiculopathy, sciatic neuropathy, and lumbar plexopathy; therefore, examination of hamstring muscles innervated by the tibial branch of the sciatic nerve as well as nontibial innervated S1 muscles should be included in the evaluation.

\section{Sciatic Neuropathy}

L4-S2 nerve roots comprise the peroneal and tibial divisions, which form the sciatic nerve, which exits the pelvis through the sciatic notch. The nerve usually passes below the piriformis muscle although occasionally the nerve or 1 of its

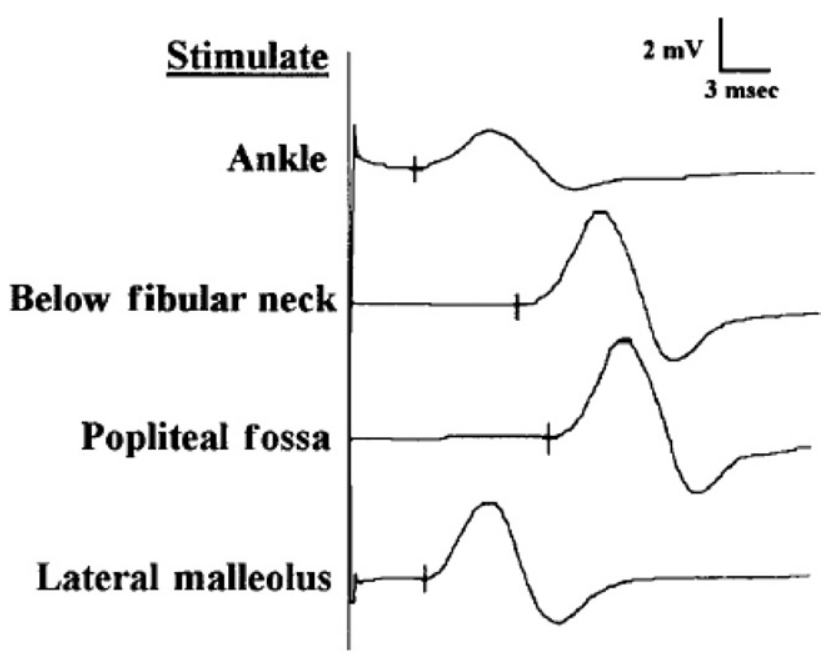

Figure 4. The presence of an accessory peroneal nerve is suspected when, during routine peroneal nerve conductions to the extensor digitorum brevis (EDB), the response obtained by stimulating at the fibular head and popliteal fossas is greater than obtained by stimulating at the ankle. An accessory peroneal nerve is confirmed by obtaining a response stimulating posterior to the lateral malleolus while recording over the EDB. (Reprinted with permission from Preston DC, Shapiro BE. Anomalous innervations. In: Preston DC, Shapiro BE. Electromyography and Neuromuscular Diseases. 2nd ed. Philadelphia, PA: Elsevier Butterworth Heinemann; 2005, 79-86) (26). 
Table 1. Nerve conduction studies in the assessment of foot drop

\begin{tabular}{lcccc}
\hline \multicolumn{1}{c}{ Lesion } & Superficial Peroneal Sensory & Sural Sensory & Tibial Motor & Peroneal Motor \\
\hline LS plexus & $\downarrow$ Ampl & $\downarrow$ Ampl & $\downarrow$ Ampl; NI CV & $\downarrow$ Ampl; NI CV \\
L5 radiculopathy & $\mathrm{Nl}$ & $\mathrm{Nl}$ & $\mathrm{Nl}$ & $\downarrow ; \mathrm{NICV}$ \\
Sciatic & $\downarrow$ & $\downarrow$ & $\downarrow ; \mathrm{NICV}$ & $\downarrow ; \mathrm{NICV}$ \\
Common peroneal & $\downarrow$ & $\downarrow$ & $\mathrm{Nl}$ & $\downarrow$ with focal block at fibular head \\
Deep peroneal & $\mathrm{Nl}$ & $\mathrm{Nl}$ & $\mathrm{NI}$ & $\downarrow$ NI CV \\
Superficial peroneal & $\downarrow$ & $\mathrm{Nl}$ & $\mathrm{Nl}$ & $\mathrm{Nl}$ \\
\hline
\end{tabular}

$\mathrm{LS}=$ lumbosacral; Ampl = amplitude; $\mathrm{NI}=$ normal; $\mathrm{CV}=$ conduction velocity; down arrow $=$ decreased.

branches may pass through or above the muscle [18]. It then courses under the gluteus maximus between the ischial tuberosity and the greater trochanter, close to the posterior aspect of the hip joint. The tibial and peroneal divisions run as distinct nerves and do not exchange fascicles along their course in the thigh. The peroneal division is more susceptible to injury than is the tibial division. This is thought to result from several mechanisms. The peroneal nerve is tethered at 2 sites, as the sciatic nerve exits the pelvis and again as it courses around the fibular head, which makes it more vulnerable to traction injury than the tibial nerve, which is not restricted distally. The peroneal nerve also contains larger fascicles with less supporting epineural tissue than the tibial division, which makes it less tolerant of external compression [19]. The tibial division innervates all of the hamstring muscles with the exception of the short head of the biceps femoris and a portion of the adductor magnus. There is no sensory branch arising from the sciatic nerve in the thigh. The sensation of the posterior thigh is carried by the posterior femoral cutaneous nerve, which arises directly from the lumbar plexus.

The sciatic nerve can be injured proximally in the pelvis or gluteal region, or less commonly, distally in the thigh. Trauma is the most common cause of proximal injuries, often accompanying fractures of the hip joint. Derangement of the sacroiliac joint can damage the sciatic nerve but often with concomitant injuries to other nerves and the plexus. The nerve is particularly susceptible to posterior hip dislocations and can be injured in operations at the hip. Incidence of sciatic neuropathy ranges from $0.05 \%$ to $1.9 \%$ [20], although electromyographic evidence of neurologic injury may be seen in up to $70 \%$ of patients after hip replacement without clinically evident weakness, with the sciatic nerve involved in most of these cases [21]. The nerve can also be injured perioperatively by supine positioning, particu- larly in very thin patients, and while in the lithotomy position [22]. Masses such as tumors, hematomas, and heterotopic ossification may cause compression of the nerve. The nerve can also be injured more distally in the thigh, often from trauma or external compression.

Sciatic neuropathy will affect the hamstring muscles, which causes weakness of hip extension and knee flexion, and weakness of muscles in the peroneal and tibial nerve distribution, with a diffusely weak foot and ankle. Incomplete injuries may be mistaken for a more distal peroneal injury due to the predilection for more severe injury to the peroneal division, as previously discussed. In addition, the tibial-innervated muscles, the hamstrings, and plantarflexors of the ankle are very powerful and subtle weakness may be more difficult to detect clinically than the less powerful dorsiflexor and evertor groups of the peroneal division. Achilles and hamstring reflexes may be diminished or absent, with the patellar reflex unaffected. Sensory abnormalities will be seen in the lower leg in the peroneal and tibial distributions. In pure sciatic injuries, posterior thigh sensation should be unaffected; however, the posterior femoral cutaneous nerve runs in close proximity to the sciatic nerve and may also be injured.

NCS in sciatic neuropathy will yield reduced amplitudes of the superficial peroneal sensory and sural sensory responses. Likewise, both tibial and peroneal motor responses will be low, without evidence of a focal conduction block at the knee. The needle examination is particularly helpful in localizing the lesion. Evidence of denervation will be seen in both the peroneal and tibial divisions and in more proximal hamstring muscles. The short head of the biceps femoris is particularly useful in situations in which the peroneal division is preferentially injured, because it is the only muscle innervated by the peroneal division while still part of the

Table 2. Needle electromyography in the assessment of foot drop

\begin{tabular}{|c|c|c|c|c|c|c|}
\hline Lesion & $\begin{array}{c}\text { Lumbar } \\
\text { Paraspinals }\end{array}$ & Gluteus Medius & $\begin{array}{l}\text { Biceps Femoris } \\
\text { (short head) }\end{array}$ & $\begin{array}{c}\text { Medial } \\
\text { Gastrocnemius }\end{array}$ & $\begin{array}{l}\text { Anterior } \\
\text { Tibialis }\end{array}$ & $\begin{array}{c}\text { Peroneus } \\
\text { Longus }\end{array}$ \\
\hline LS plexus & $\mathrm{NI}$ & Abnl & Abnl & Abnl & Abnl & Abnl \\
\hline Sciatic & $\mathrm{NI}$ & $\mathrm{NI}$ & Abnl & Abnl & Abnl & Abnl \\
\hline Common peroneal & $\mathrm{NI}$ & $\mathrm{NI}$ & $\mathrm{NI}$ & $\mathrm{NI}$ & Abnl & Abnl \\
\hline Deep peroneal & $\mathrm{NI}$ & $\mathrm{NI}$ & $\mathrm{NI}$ & $\mathrm{NI}$ & Abnl & $\mathrm{NI}$ \\
\hline Superficial peroneal & $\mathrm{NI}$ & $\mathrm{NI}$ & $\mathrm{NI}$ & $\mathrm{NI}$ & $\mathrm{NI}$ & Abnl \\
\hline
\end{tabular}

$\mathrm{LS}=$ lumbosacral; $\mathrm{NI}=$ normal; $\mathrm{Abnl}=$ abnormal. 


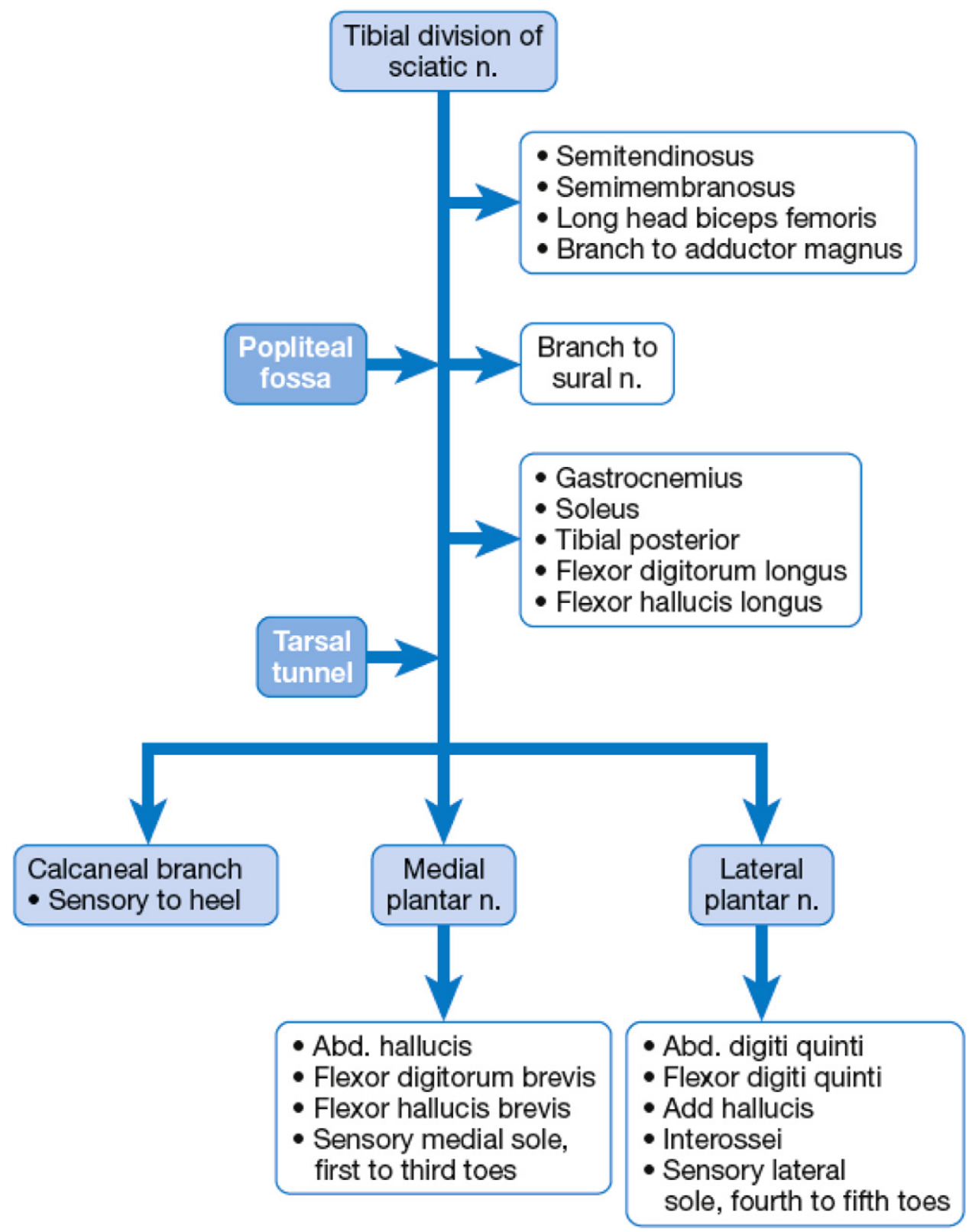

Figure 5. Tibial nerve (Reprinted with permission from Craig A, Richardson J. Rehabilitation of patients with neuropathies. In: Braddom RL, ed. Physical Medicine and Rehabilitation. Philadelphia, PA: Elsevier; 2011, 1065-1095) (26).

sciatic nerve proper. Examination of other gluteal muscles and paraspinal muscles should be performed to exclude a more proximal plexus injury or L5-S1 radiculopathy.

\section{Superior and Inferior Gluteal Nerves}

The superior gluteal nerve derives from the L4-S1 nerve roots and passes through the sciatic notch above the piriformis muscle. It provides innervation to the gluteus medius and minimus muscles and to the tensor fascia lata. The inferior gluteal nerve arises from the L5-S2 nerve roots and supplies only the gluteus maximus muscle. Both nerves are rarely injured without iatrogenic cause. Up to three-fourths of patients will demonstrate subclinical electromyography changes in these muscles after hip replacement surgery [23]. These nerves, particularly the superior gluteal, can be injured by poorly placed intramuscular injections. The gluteal nerves may be compressed by pelvic masses, such as with colorectal cancer or iliac artery aneurysm [24].

Superior gluteal nerve injury presents with atrophy and weakness of the gluteus medius, minimus, and tensor fascia 
lata muscles, with weakness of hip abduction and external rotation. Patients will demonstrate a Trendelenburg gait, with pelvic tilt toward the strong side. Inferior gluteal injury will result in atrophy of the gluteus maximus and weakness of leg extension. There should be no sensory changes unless there is concomitant injury of the posterior femoral cutaneous nerve, although deep aching pain may be a feature, particularly with compressive tumors. Physical examination findings may be subtle in milder lesions and may be difficult to assess, particularly in the setting of a previous operation.

The finding of electrodiagnostic abnormalities on needle study of the gluteal muscles can be helpful in detecting gluteal neuropathies. Other L5 and S1 as well as paraspinal muscles should be sampled to rule out sciatic, plexus, or radicular lesions. There is no NCS available to assess the gluteal nerves.

\section{CONCLUSION}

Mononeuropathies of the nerves to the lower limbs are common and can have significant effects on mobility and function. These injuries often accompany trauma and surgical and medical procedures. Particularly in cases of severe trauma, the initial nerve injury may be masked by the musculoskeletal injury and may manifest only after delayed return of function. A thorough understanding of the anatomy of the nerves of the lower extremity, their points of vulnerability, and functional role is valuable in identifying injury. The role of electrodiagnosis is to confirm and localize the neurologic injury, provide information on the severity and chronicity of the injury, and exclude other causes of neurologic deficit, such as plexopathy or radiculopathy.

\section{CLINICAL PEARLS}

- Prolonged positioning in the lithotomy position may compromise the femoral nerve at the inguinal ligament due to sharp angulation of the nerve in the flexed, abducted, and externally rotated position Obturator nerve (and, less commonly, the common peroneal nerve) can also be injured with the lithotomy position.

- The femoral motor NCS are uncomfortable due to longer duration stimulation and may require comparison with the opposite side. Obtaining a compound action potential of at least $50 \%$ of the other side suggests good prognosis for recovery within 1 year.

- Isolated injury to the infrapatellar branch of the saphenous nerve may present with medial knee pain that may be mistaken for medial knee compartment pathology.

- The obturator nerve resides anterior to the sacroiliac joint and may be infrequently injured from pelvic trauma. However, the extent of these pelvic trauma injuries will usually also involve other nerves, the plexus, or spinal roots.
- Ultrasound can be used to identify the lateral femoral cutaneous nerve location at the ASIS and may improve the ability to obtain sensory nerve action potentials.

- In addition to direct compression mechanism at the fibular head, severe flexion and inversion sprains at the ankle can also cause traction injuries due to tearing of the vasa nervorum of the common peroneal nerve.

- The short head of the biceps femoris will help in localizing lesions to above or below the knee, because this muscle is the only peroneal-innervated muscle above the knee. Incomplete sciatic nerve injuries may be mistaken for a more distal peroneal injury due to the predilection for more severe injury to the peroneal division.

- Tarsal tunnel syndrome presents with numbness and pain in the sole of the foot, often sparing the calcaneal branch to the heel. Needle electromyography abnormalities in the first dorsal interosseous reflect injury of the lateral plantar branch, and abnormalities in the adductor hallucis reflect injury of the medial plantar branch. Needle comparisons to the corresponding muscles of the contralateral side should be performed to rule out early peripheral neuropathy etiologies for the foot intrinsic muscle abnormalities.

\section{REFERENCES}

1. Moore A, Stringer M. Iatrogenic femoral nerve injury: A systematic review. Surg Radiol Anat 2011;33:649-658.

2. Bohrer JC, Walter MD, Park A, et al. Pelvic nerve injury following gynecologic surgery: A prospective cohort study. Am J Obstet Gynocol 2009;201:531-537

3. Ducic I, Delton L, Larson EE. Treatment concepts for idiopathic and iatrogenic femoral nerve mononeuropathy. Ann Plast Surg 2005;55: 397-401.

4. Kuntzer T, van Melle G, Regli F. Clinical and prognostic features in unilateral femoral neuropathies. Muscle Nerve 1997;20:205-211.

5. Stewart JD, ed. Focal Peripheral Neuropathies. 3rd ed. Philadelphia, PA: Lippincott Williams \& Wilkins; 2000.

6. Nair UR, Griffiths G, Lawson RA. Postoperative neuralgia in the leg after saphenous vein coronary artery bypass graft: A prospective study. Thorax 1988;43:41-43.

7. Sorenson EJ, Chen JJ, Daube JR. Obturator neuropathy: Causes and outcome. Muscle Nerve 2002;25:605-607.

8. Wagner MA, Warner DO, Harper CM, et al. Lower extremity neuropathies associated with lithotomy positions. Anesthesiology 2000;93: 938-942.

9. Warfield CA. Obturator neuropathy after forceps delivery. Obstet Gynecol 1984;64:47S-48S.

10. Bradshaw C, McCrory P, Bell S, Brukner P. Obturator neuropathy: A cause of chronic groin pain in athletes. Am J Sports Med 1997;25:402 408.

11. Ivins GK. Meralgia parasthetica, the elusive diagnosis: Clinical experience with 14 adult patients. Ann Surg 2000;232:281-286.

12. Shapiro BE, Preston DC. Entrapment and compression neuropathies. Med Clin N Am 2009;93:285-315.

13. Langueny R, Deliac MM, Deliac P, et al. Diagnostic and prognostic value of electrophysiologic tests in meralgia parasthetica. Muscle Nerve 1991;14:51-56.

14. Boon AJ, Bailey PW, Smith J, et al. Utility of ultrasound-guided surface electrode placement in lateral femoral cutaneous nerve conduction studies. Muscle Nerve 2011;44:525-530. 
15. Preston DC, Shapiro BE. Peroneal nerve palsy. In: Preston DC, Shapiro BE, eds. Electromyography and Neuromuscular Disorders: ClinicalElectrophysiologic Correlations. 2nd ed. Philadelphia, PA: Elsevier; 2005, 343-354.

16. Stewart JD. Foot drop: Where, why and what to do? Pract Neurol 2008 8:158-169.

17. Takakura $Y$, Kumai $T$, Takaoka $T$, Tamai $S$. Tarsal tunnel syndrome caused by coalition associated with a ganglion. J Bone Joint Surg Br 1998;80:130-133.

18. Benzon HT, Katz JA, Benzon HA, Iqbal MS. Piriformis syndrome: Anatomic considerations, a new injections technique, and a review of the literature. Anesthesiology 2003;98:1442-1448.

19. Sunderland $S$. The relative susceptibility to injury of the medial and lateral popliteal division of the sciatic nerve. Br J Surg 1953;411:300302.

20. Brown GD, Swanson EA, Nercessian OA. Neurologic injuries after total hip arthroplasty. Am J Orthop 2008;37:191-197.
21. Weber ER, Daube JR, Convenry MB. Peripheral neuropathies associated with total hip arthroplasty. J Bone Joint Surg Am 1976;58:66-69.

22. Warner MA, Warner DO, Harper M, Schroeder DR, Maxson PM. Lower extremity neuropathies associated with lithotomy position. Anesthesiology 2000;93:938-942.

23. LaBan MM, Meerschaert JR, Taylor RS. Electromyographic evidence of inferior gluteal nerve compromise: An early representation of recurrent colorectal carcinoma. Arch Phys Med Rehabil 1982;63:33-35.

24. Abitbol JJ, Gendron D, Laurin CA, Beaulieu MA. Gluteal nerve damage following total hip arthroplasty: A prospective analysis. J Arthroplasty 1990;5:319-322.

25. Craig A, Richardson J. Rehabilitation of patients with neuropathies. In: Braddom RL, ed. Physical Medicine and Rehabilitation. Philadelphia PA: Elsevier; 2011, 1065-1095.

26. Preston DC, Shapiro BE. Anomalous innervations. In: Preston DC, Shapiro BE. Electromyography and Neuromuscular Diseases. 2nd ed. Philadelphia, PA: Elsevier Butterworth Heinemann; 2005, 79-86. 